\title{
Síndrome de Dress: reacción alérgica tras la colocación de un catéter peritoneal
}

\author{
Natalia Formento-Marín, María José Cintora-Quero, María Soriano-Angulo, Raúl Pablo-Gormaz, Belén \\ Campos-Gutiérrez \\ Servicio de Enfermedad Renal Crónica Avanzada y Diálisis Peritoneal. Hospital Universitario Miguel Servet. \\ Zaragoza. España
}

Como citar este artículo: Formento-Marín N, Cintora-Quero MJ, Soriano-Angulo M, Pablo-Gormaz, R, Campos-Gutiérrez B. Síndrome de Dress: reacción alérgica tras la colocación de un catéter peritoneal.

Enferm Nefrol. 2020 Abr-Jun;23(2):207-10

\section{Resumen}

Caso clínico: Paciente que inició un Síndrome de Dress tras la colocación del catéter peritoneal. En la literatura se han reportado un número muy escaso de casos de reacción alérgica al catéter peritoneal e inicialmente se sospechó como causa un antibiótico profiláctico utilizado en el quirófano, pero tras sufrir en su domicilio una exacerbación de la sintomatología a la semana de la implantación, se planteó la posibilidad de que el catéter fuera el origen del cuadro, por lo que el paciente ingresó para monitorización y administración de medicación con una mejoría clínica significativa. El Plan de Cuidados se centraba en la resolución aguda del proceso alérgico y asistir al paciente en la técnica hasta que pudo realizarla autónomamente. Finalmente, el cuadro clínico se atribuyó a un Síndrome de Dress provocado por alopurinol.

Conclusiones: Tras una revisión bibliográfica y ante la falta de evidencia, se decidió no retirar el catéter y tratar el cuadro, que se solucionó, pudiendo iniciar el paciente la técnica en domicilio de forma autónoma.

PALABRAS CLAVES: alergia; catéter; diálisis peritoneal; síndrome de Dress.

\section{Correspondencia:}

Natalia Formento Marín

Email: natalia.formento7@gmail.com
Dress syndrome: allergic reaction after placement of a peritoneal catheter

\section{Abstract}

Clinical case: Patient suffering from Dress Syndrome after placement of the peritoneal catheter. A very few cases of allergic reaction to the peritoneal catheter have been reported in the literature. Initially, the use of a prophylactic antibiotic used in the operating room was suspected as a cause, but after suffering an exacerbation of symptoms one week after implantation at the patient's home, the possibility that the catheter was the origin of the condition was raised. Then, the patient was admitted for monitoring and administration of medication with significant clinical improvement. The care plan was focused on the acute resolution of the allergic process and on assisting the technique until the patient was able to perform it autonomously. Finally, the clinical picture was attributed to a Dress Syndrome caused by allopurinol.

Conclusions: After a bibliographic review and in the absence of evidence, it was decided not to remove the catheter and treat the clinical picture, which was resolved, and the patient could start the technique at home autonomously.

Keywords: allergy; catheter; peritoneal dialysis; Dress syndrome. 


\section{Introducción}

La reacción alérgica tras la inserción del catéter peritoneal es un evento poco estudiado en la literatura científica. Para encontrar los primeros casos, nos tenemos que remontar a 1985, donde se publica el primer estudio donde se describe un episodio de eosinofilia y rash cutáneo en la zona del orificio de salida ${ }^{1}$ y el siguiente estudio 26 años más tarde ${ }^{2}$, donde se reporta un caso de eosinofilia con hiperpigmentación macular y rash cutáneo con prurito de afectación sistémica que se resolvió tras la retirada del catéter peritoneal. En ambos casos el modelo utilizado fue el mismo dispositivo que el caso que presentamos en nuestro estudio, un catéter tipo Tenchkhoff compuesto principalmente por silicona.

A pesar de haber encontrado pocas publicaciones científicas que describan episodios con sintomatología alérgica relacionados con la inserción del catéter peritoneal, sí que se han descrito casos similares relacionados a otros dispositivos fabricados con silicona ${ }^{2}$. Sin embargo, no podemos confirmar por casos únicos la posibilidad de que la silicona del catéter peritoneal produzca una reacción inmunitaria, a pesar de que puede contener aditivos químicos con la capacidad de provocar este tipo de reacción 3 .

\section{Presentación del caso}

Paciente de 60 años de origen chino, con antecedentes clínicos de insuficiencia cardiaca por insuficiencia aórtica severa, enfermedad renal crónica avanzada de etiología desconocida, hipertensión, anemia nefrógena e hiperuricemia. En tratamiento con eritropoyetina, paricalcitol, amlodipino, atorvastatina, bisoprolol, ácido acetilsalicílico y alopurinol. Sin alergias medicamentosas conocidas hasta el episodio descrito.

A las 8 horas de la colocación del catéter peritoneal, el paciente presenta un eritema pruriginoso generalizado en tronco, abdomen y extremidades superiores e inferiores, con eosinofilia de $28,7 \%$. En un primer momento, se sospecha su relación con la administración de cefazolina como antibiótico profiláctico antes de la cirugía debido a la secuencia temporal. Durante el ingreso se instaura tratamiento con corticoides y dexclorfeniramina intravenoso con mejoría clínica por lo que se procede al alta a domicilio siendo derivado a las consultas de alergia para estudio.

A la semana, al iniciar el entrenamiento en diálisis peritoneal en la consulta de enfermería, el paciente acude con una exacerbación de la clínica, presentando una erupción eritemato-descamativa generalizada, con prurito, grietas en pies y manos y edemas generalizados (Figuras $\mathbf{1}$ y 2 ).

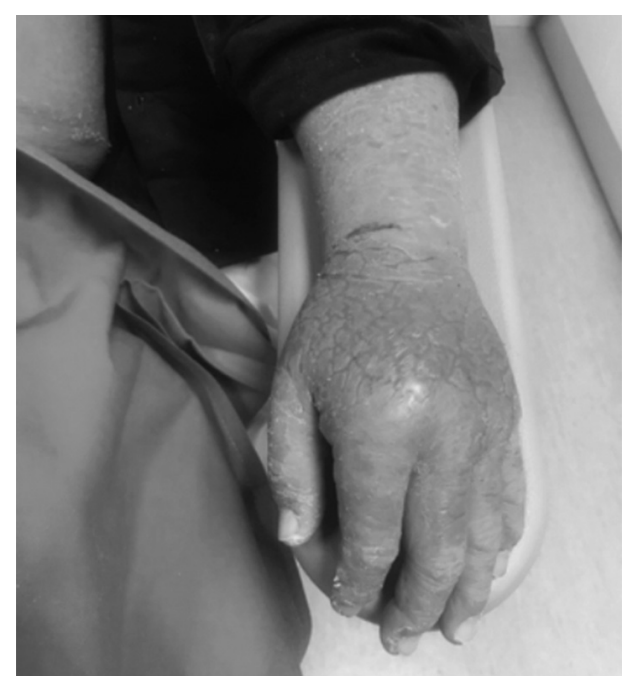

Figura 1. Mano izquierda del paciente tras la exacerbación. Extremidad muy edematizada, eritematosa, con grietas en zona dorsal y palmar con dolor y prurito.

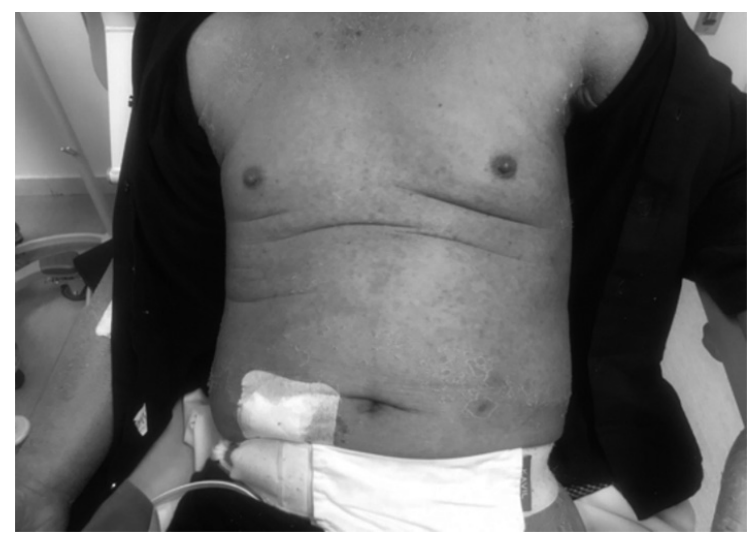

Figura 2. Reacción eritematosa-descamativa por tronco. El paciente presentaba una reacción sistémica en tronco, extremidades superiores e inferiores, espalda y cara con prurito.

Ante esta situación, se decide suspender el entrenamiento ante la incapacidad del paciente para la realización de la técnica. Se contacta con el servicio de dermatología para su valoración y se decide su ingreso para seguimiento. Ante el empeoramiento de la clínica y la secuencia temporal de aparición, nos planteamos la posibilidad de que el paciente haya desarrollado una dermatitis por contacto al catéter peritoneal. Se realiza una revisión bibliográfica y ante la insuficiente evidencia científica encontrada y con la posibilidad de monitorizar al paciente ingresado se decide no retirar el catéter peritoneal y seguir su evolución. 
Tras la valoración por dermatología, el paciente es diagnosticado de un síndrome de Dress (Drug Rash with Eosinophilia and Systemic Symptoms), una reacción a fármacos con eosinofilia y síntomas sistémicos que se relaciona con el tratamiento con alopurinol, por lo que se decide la retirada de esta medicación pautada desde hace dos semanas y tras tratamiento con corticoesteroides y antihistamínicos intravenosos y fomentos de sulfato de zinc tópicos el paciente presenta una mejoría notable en $24 \mathrm{~h}$ con resolución de la clínica, por lo que se decide dar de alta a domicilio con un pauta de corticoides orales y tratamiento tópico (Figura 3).

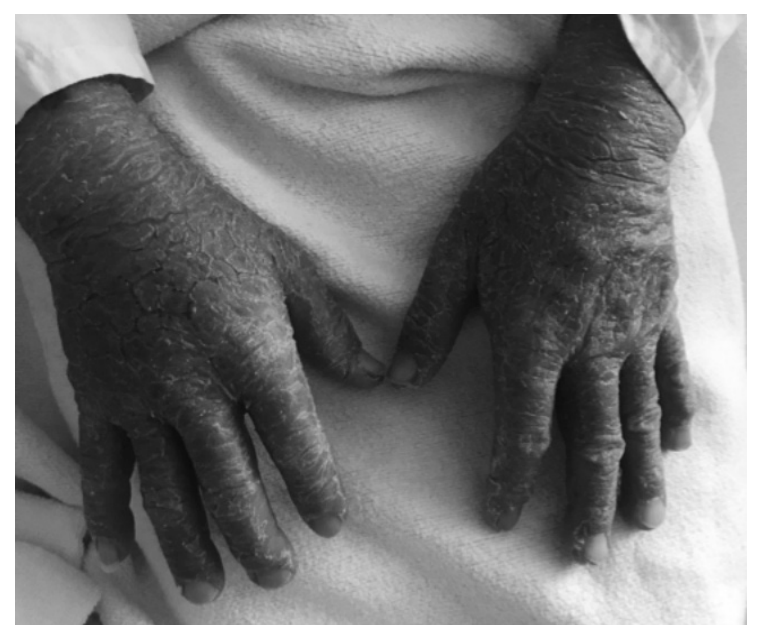

Figura 3. Manos del paciente a las $24 \mathrm{~h}$ del ingreso, tras la retirada del alopurinol y la administración de tratamiento. Mejoría de los edemas, continua con importante descamación y grietas.

Por parte del servicio de diálisis peritoneal se reanudó el entrenamiento con el paciente, que precisó una mayor duración hasta que las lesiones de las manos le permitieron realizar la técnica autónomamente llevando a cabo una adecuada técnica aséptica con el uso de solución hidroalcohólica.

\section{Plan de cuidados de enfermería}

Se realiza una valoración inicial identificando todos los problemas potenciales de salud que pueden ser potencialmente tratados mediante el plan de cuidados, basado en los patrones funcionales de Marjory Gordon. A continuación, se detallan aquellos patrones valorados:

\section{Patrón 1: Percepción-manejo de la salud}

Paciente sin alergias medicamentosas conocidas hasta la fecha, original de china, con apoyo familiar para entrenamiento en terapia renal sustitutiva domiciliaria, no hábitos tóxicos, ingresado hace dos semanas en hospital por una insuficiencia aórtica severa con insuficiencia renal crónica de origen desconocido, sin antecedentes de patologías previas conocidas.

\section{Patrón 2: Nutricional-metabólico}

Aumento de peso significativo respecto al último control debido a la presencia de edemas con importante fóvea en extremidades inferiores, superiores y cara. Presenta lesiones cutáneas en manos, tórax y cara con una erupción eritemato-descamativa generalizada, con prurito y grietas en pies y manos. Diuresis de $1000 \mathrm{ml}$ en 24h.

\section{Patrón 3: Eliminación}

Adecuado hábito intestinal sin estreñimiento. Sin incidencias en el funcionamiento del catéter.

\section{Patrón 4: Actividad}

Independiente para las actividades básicas de la vida diaria (ABVD) y con incapacidad para la realización de actividades instrumentales de la vida diaria (AIVD) debido a la presencia de grietas en manos que le impiden actividades como abrocharse los botones o realizar la terapia domiciliaria. No realiza ejercicio habitualmente.

\section{Patrón 6: Cognitivo-perceptual}

Orientado en espacio-tiempo. Hipermetropía corregida con lentes, sin otras alteraciones sensoriales. Déficit de comunicación debido a las dificultades para entender y hablar español. Con prurito y dolor por las lesiones cutáneas. Sin dificultades para el aprendizaje.

\section{Patrón 8: Rol-relaciones}

Vive con su mujer y trabajan en una tienda. Adecuado soporte familiar con un hijo y sobrina que actúan de mediadores con el equipo para poder comunicarnos adecuadamente con el paciente.

Tras la identificación de problemas potenciales de salud realizado por la enfermera, se establece un plan de cuidados para su abordaje siguiente la taxonomía NANDANOC-NIC (4):

\section{Diagnósticos / NANDA:}

00004: Riesgo de infección, relacionado con la alteración de la integridad de la piel.

00078: Gestión ineficaz de la salud, relacionado con la dificultad para gestionar un régimen terapéutico complejo y manifestado por el fracaso al incluirlo en la vida diaria. 


\section{Objetivos / NOC:}

1101-Integridad tisular: piel y membranas mucosas. Puntuación inicial: 2, puntuación diana: 4. Indicadores propuestos para su evaluación: 110115 - Lesiones cutáneas. Valor escala: 2; 110119 - Descamación cutánea. Valor escala: 2; 110121 - Eritema. Valor escala: 2. Escala de medición: grave 1, sustancial 2, moderado 3, leve 4 y ninguno 5).

2302-Aclaración de toxinas sistémicas: diálisis. Puntuación inicial: 2-3, puntuación diana: 4. Indicadores propuestos para su evaluación: 230225-Aclaramiento de creatinina. Valora escala: 3; 230212 - Tasa de reducción de urea. Valor escala: 3. Escala de medición: desviación grave del rango normal 1 , desviación sustancial del rango normal 2, desviación moderada del rango normal 3, desviación leve del rango normal 4, sin desviación del rango normal 5 .

\section{Intervenciones / NIC:}

6410-Manejo de la alergia: Suministrar medicamentos para reducir o minimizar una respuesta alérgica; Enseñar al paciente/progenitor a que evite sustancias alérgicas, según corresponda.

2150-Terapia de diálisis peritoneal: Enseñar el procedimiento al paciente que requiera diálisis domiciliaria.

\section{Discusión}

Los casos encontrados en la bibliografía son escasos teniendo en cuenta la cantidad de catéteres peritoneales utilizados. Las reacciones encontradas en la bibliografía coinciden con las propias de una reacción alérgica y con las de nuestro paciente, eosinofilia, rash cutáneo y prurito, aunque la gravedad del cuadro de nuestro caso es mayor, con una afectación sistémica. El tipo de catéter utilizado en todos los casos es el mismo, silicona como material principal. Pese a ello, ante la falta de evidencia y el escaso número de casos, se decidió esperar y valorar otras posibles causas, finalmente se resolvió el episodio sin necesidad de retirar el catéter y el paciente pudo mantener la técnica domiciliaria elegida.
La retirada del catéter tempranamente no nos hubiera permitido discernir si el episodio se debió a la administración del antibiótico profiláctico antes de la colocación del catéter peritoneal o al propio catéter.

El plan de cuidados elaborado tras la valoración inicial del paciente se centraba en la resolución de la reacción alérgica del paciente y posteriormente en la adecuada asistencia y formación en la técnica de diálisis peritoneal hasta que el paciente pudo llevarla a cabo en su domicilio. Los resultados obtenidos en la evaluación fueron los esperados.

Recibido: 27-02-20

Revisado: 15-03-20

Modificado: 03-04-20

Aceptado: 25-04-20

\section{Bibliografía}

1. Kurihara S, Tani Y, Tateishi K, Yuri T, Kitada H, Sugishita $\mathrm{N}$ et al. Allergic eosinophilic dermatitis due to silicone rubber: a rare but troublesome complication of Tenckhoff catheter. Perit Dial Int 1985; 5:65-7.

2. Patel UO, Fox SR, Moy JN, Korbet SM. Pruritic rash and eosinophilia in a patient receiving peritoneal dialysis. Semin Dial. 2011;24(3):338-40.

3. Curtis $\mathrm{J}$, Klykken P. Comments on a case report of a Tenckhoff catheter allergy. Semin Dial. 2011;24(6):686-7.

4. Herramienta online para la consulta y diseño de Planes de Cuidados de Enfermería. [Internet]. NNNConsult. Elsevier; 2015 [consultado 01 may 2020]. Disponible en: http://www.nnnconsult.com/.

Este artículo se distribuye bajo una Licencia Creative Commons Atribución-NoComercial 4.0 Internacional. https://creativecommons.org/licenses/by-nc/4.0/

Open Access (c) () () 